\title{
Infected Esophageal Duplication Cyst Masquerading as Pericarditis
}

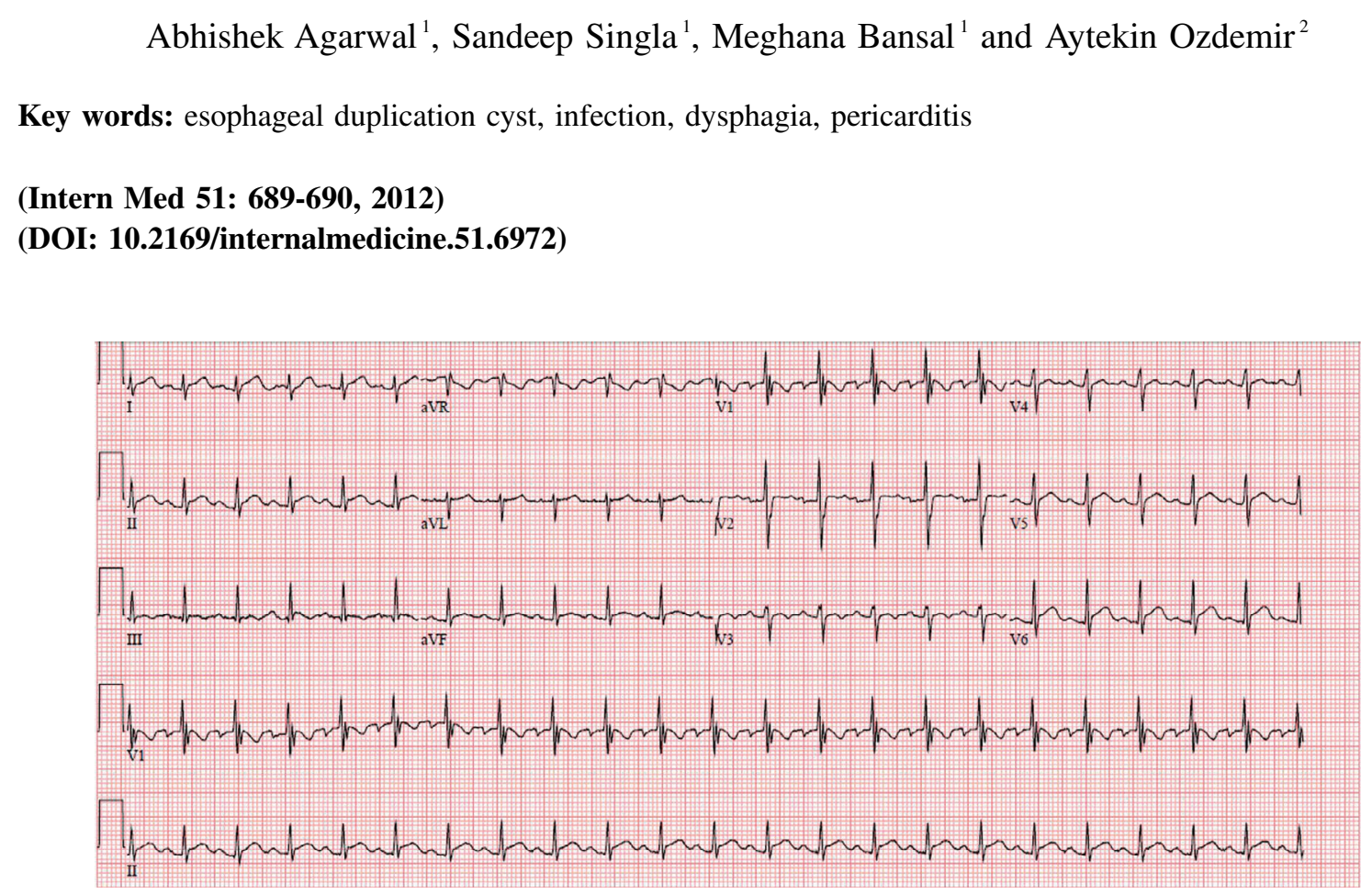

Picture 1.

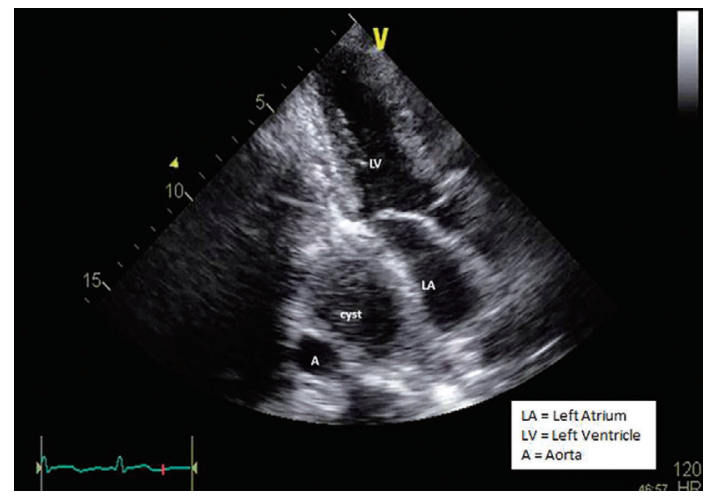

Picture 2.

A 24-year-old woman presented with dysphagia, chest pain and fever for three days. On examination, she had epigastric tenderness. Leukocyte count was 11,000 cells $/ \mu \mathrm{L}$ with $82 \%$ neutrophils, with normal serum troponins and amylase. Electrocardiogram showed diffuse PR depression and ST elevation suggestive of pericarditis (Picture 1). Echocardiogram showed a cystic mass partially compressing the left atrium (Picture 2). Computed tomography showed a $6 \mathrm{~cm}$ peripherally enhancing cystic mass (arrow) in the lower chest consistent with esophageal duplication cyst (Picture 3). Thoracotomy revealed a large, indurated mass between the aorta and the posterior surface of the pericardium engulfing the esophagus which was resected. Abscess cultures grew methicillin-sensitive Staphylococcus aureus which was treated with antibiotics.

Esophageal duplication cysts are congenital anomalies that are often detected incidentally on chest radiographs. Complications include infection, bleeding, rupture and mass effect (1). Surgical resection is generally required for cysts that cause symptoms.

The authors state that they have no Conflict of Interest (COI).

${ }^{1}$ Department of Internal Medicine, University of Arkansas for Medical Sciences, USA and ${ }^{2}$ Department of Surgery, University of Arkansas for Medical Sciences, USA

Received for publication November 21, 2011; Accepted for publication November 23, 2011

Correspondence to Dr. Agarwal Abhishek, aagarwal@uams.edu 
Intern Med 51: 689-690, 2012 DOI: 10.2169/internalmedicine.51.6972

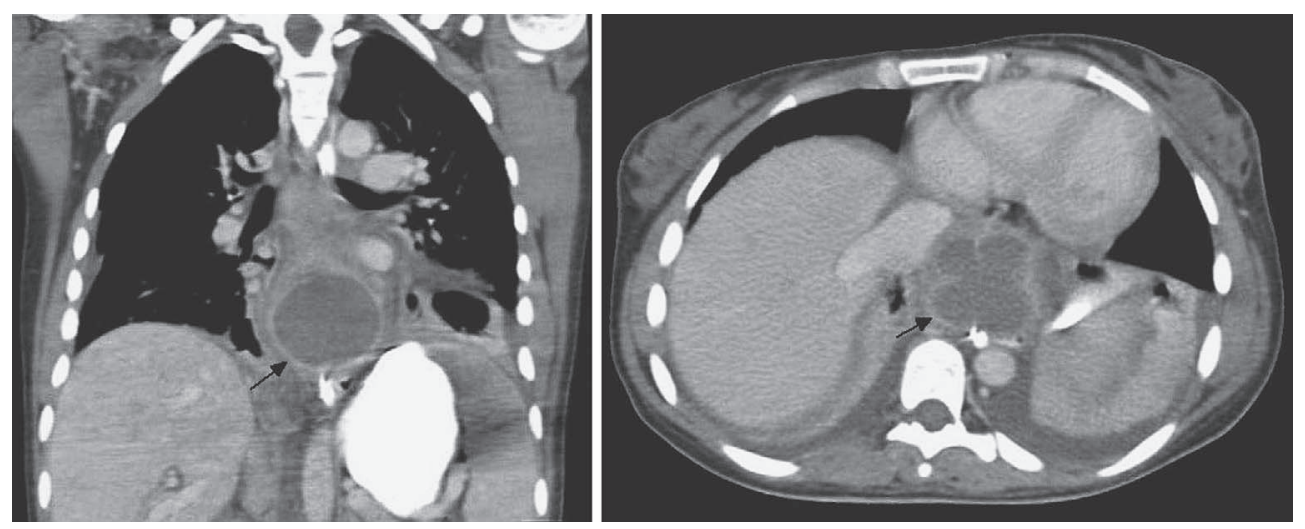

Picture 3.

\section{Reference}

1. Javan NG, Debnath J, Kumar A, Das CJ. Oesophageal duplication cyst: an unusual cause of retrosternal pain and dysphagia in an adult. Singapore Med J 49: e242-e244, 2008.

\footnotetext{
(C) 2012 The Japanese Society of Internal Medicine http://www.naika.or.jp/imindex.html
} 